\title{
Sex Hormones as Cognitive Enhancers?
}

\author{
Francisco Núñez ${ }^{2}$ - María J. Maraver ${ }^{3,4} \cdot$ Lorenza S. Colzato $^{1,2}$
}

Received: 20 January 2019 / Accepted: 15 October 2019 / Published online: 10 December 2019

(C) The Author(s) 2019

\begin{abstract}
Understanding the differences in the way women and men think has made headway thanks to experiments showing how sex hormones influence cognitive capacities. Masculine and feminine sex hormones (androgens and estrogens, respectively) affect cognition in different ways and may account for some of the gender differences in cognitive abilities, allowing men and women to perform better in certain cognitive tests. In this opinion article, we discuss studies addressing differences in cognitive functions between males and females and the underlying neural substrates, as well as the effects of sex hormone supplementation. Even though some studies on patients receiving exogenous sex hormones showed gender differences that emerge at group levels on a few cognitive tasks, it is not yet clear whether these differences can be partially attributed to hormonal causes. Supplementation of female estrogen can enhance verbal skills, whereas masculine androgen can increase performance in mathematical and visuospatial tasks. Studies of the administration of exogenous sex hormones have allowed further insight into the use of sex hormones as possible cognitive enhancers.
\end{abstract}

Keywords Testosterone $\cdot$ Estrogen $\cdot$ Gender $\cdot$ Cognitive enhancement

\section{Introduction}

Research has shown that there are no indications of large sex differences in general intelligence but that each sex has advantages in specific cognitive tasks, often with small effect sizes (Hyde 2016): on average, women excel in verbal cognition tasks such as fluency, vocabulary, verbal memory, and verbal learning, as well as in fine motor dexterity, and perceptual speed tasks; while men, in turn, show an average advantage in visual memory, visuospatial ability, and math (Hyde 2014; Vidal et al. 2006). Among others, part of what might influence these cognitive differences is sex hormones (Miller and Halpern 2014). Sex

Lorenza S. Colzato

lorenza.colzato@uniklinikum-dresden.de

1 Cognitive Neurophysiology, Department of Child and Adolescent Psychiatry, Faculty of Medicine, TU Dresden, Dresden, Germany

2 Department of Cognitive Psychology, Institute of Cognitive Neuroscience, Faculty of Psychology, Ruhr University Bochum, Dresden, Germany

3 Cognitive Psychology Unit and Leiden Institute for Brain and Cognition, Leiden University, Leiden, The Netherlands

4 Faculty of Psychology \& Research Center for Psychological Science, University of Lisbon, Lisbon, Portugal hormones are released from males and female gonads (Frick et al. 2015), and although its concentration in the bloodstream is distributed differently across genders, it is evident that estrogen levels are higher in women than men, while testosterone levels are higher in men than in women. A key question that still needs to be carefully investigated is to what extent cognitive advantages depend on sex hormones. As pointed out by Colzato and Hommel (2014), the current research on the role of sex hormones in cognition has been mainly "effect"-driven, showing that sex hormones can impact cognitive functions; however, this is without explaining the underlying mechanism of how they modulate cognitive processes.

In this opinion article, research on the cognitive differences between healthy male and female humans is discussed, and the mechanisms linking sex hormones and cognition are briefly described. Finally, we examine the effects of the administration of exogenous hormones on human cognitive capacities.

Research involving people who are receiving hormone replacement therapy due to illness or menopause-related symptoms and of those transsexual men and women taking corrective hormones can extend the theoretical understanding of hormonal mechanisms, and it opens a promising field of research by evaluating the possible use of sex hormones as cognitive enhancers. 


\section{Gender Differences}

Let us first examine the most salient preexisting (without supplementation of sex hormones) cognitive abilities in which healthy females and males might differ.

\section{Females}

Cognitive advantages of females are most evident in verbal cognition. Several studies have shown a significant advantage of females over males in verbal tasks (i.e., tests that require engaging with, manipulating, or using verbal material, such as reading and writing), during childhood and adolescence (Scheiber et al. 2015). For example, a study examining speed in phonetic processing showed that women were significantly faster and more accurate in identifying alphabetical sequences than men (Majeres 1997). Another study showed females to be more apt to identify pseudohomophones correctly (i.e., nonwords that are pronounced identically to a real word, such as heir (nonword) and air (real word)), demonstrating better pre-lexical and lexical processing (Majeres 1999). The cognitive advantages of females in verbal cognition, however, are regarded as small in comparison to other biological and environmental effectors, and the dissimilarities diminish with age (Ardila et al. 2011). Recently, Scheuringer et al. (2017) found that significant sex differences in verbal fluency were revealed only when subjects were explicitly instructed to switch between categories, which boosted the number of switches in women. Accordingly, the authors have suggested the importance to take into account the role of strategies and instructions given to males and females when investigating sex differences in verbal fluency. Along these lines, Pletzer et al. (2017) found some evidence suggesting that gender differences in cognition might be due to the fact that females and males adopt or have acquired different cognitive processing styles: males seem to be more oriented toward holistic stimulus aspects (global processing), while female approaches are more focused toward stimulus details (local processing).

\section{Males}

The main male advantage in cognition has been seen in visuospatial abilities (i.e., skills that require to represent, analyze, and mentally manipulate objects). Differences with the largest effect size have been reported in mental rotation tasks (Miller and Halpern 2014). In an example of this type of task, a figure is presented from one point of view, and four other (rotated) points of view are displayed next to it, with two of them being from the same figure and two being from a mirrored figure. The time it takes the participant to report which images belong together is measured, where faster responses imply better performance, and men normally outperform women in this type of tasks (Miller and Halpern 2014). Other studies have shown men to be slightly, yet statistically better than women in mathematical problem-solving. Kaufman et al. (2009) calculated that in standardized tests, men scored " 4.1 points $(0.27 \mathrm{SD})$ higher than women on math, whereas women outscored men by 8.8 points $(0.59 \mathrm{SD})$ on writing". It is still under debate, however, how much of this difference is biological in nature and how much it is societal-cultural, given that still tends to be more men than women in technological and math-intensive careers (Yang and Barth 2015).

\section{Mechanism of Action}

Chemically, progesterone is the precursor steroid hormone for the synthesis of androgens like testosterone (the primary male sex hormone) and estrogens (the main female sex hormone; see Fig. 1). Androgens are the primary precursor for natural estrogens, and what differentiates males and females is the number of enzymes that deal with these androgens converting them into estrogen or into testosterone (Frick et al. 2015). Moreover, though they are notably produced in the gonads, sex hormones can be synthesized within the brain (Frick et al. 2015). Further, it is important to keep in mind that estradiol precursors can also be produced by fat cells and that small amounts of estradiol are also produced in the brain (Frick et al. 2015).

\section{Estrogen}

Mainly based on animal literature, it is known that estrogen seems to promote cognitive functioning via the activation of estrogen receptor ER- $\alpha$ and ER- $\beta$ in cognitive relevant brain areas, such as the prefrontal cortex and the hippocampus (Hara et al. 2015; Tuscher et al. 2016). Estrogen facilitates the synthesis of neurotrophins (Milne et al. 2014), helps the brain coping with stress and inflammation (Luine 2014), and modulates the signaling of several neurotransmitters, above all, dopamine (Sinclair et al. 2014). After estrogen crosses the blood-brain barrier, it is transformed into cathecol estrogen, which is supposed to inhibit the catechol Omethyltransferase (Ball et al. 1972), an enzyme responsible for the degradation of dopamine in prefrontal cortex (PFC), a crucial area for the way we control our thoughts and goaldirected behavior (Miller (2000). Related to dopamine, Colzato and Hommel (2014) suggested that estrogen might modulate cognitive processes driven by this neurotransmitter. Consistent with this idea, previous studies have shown that gender differences in dopaminergic-driven cognitive 


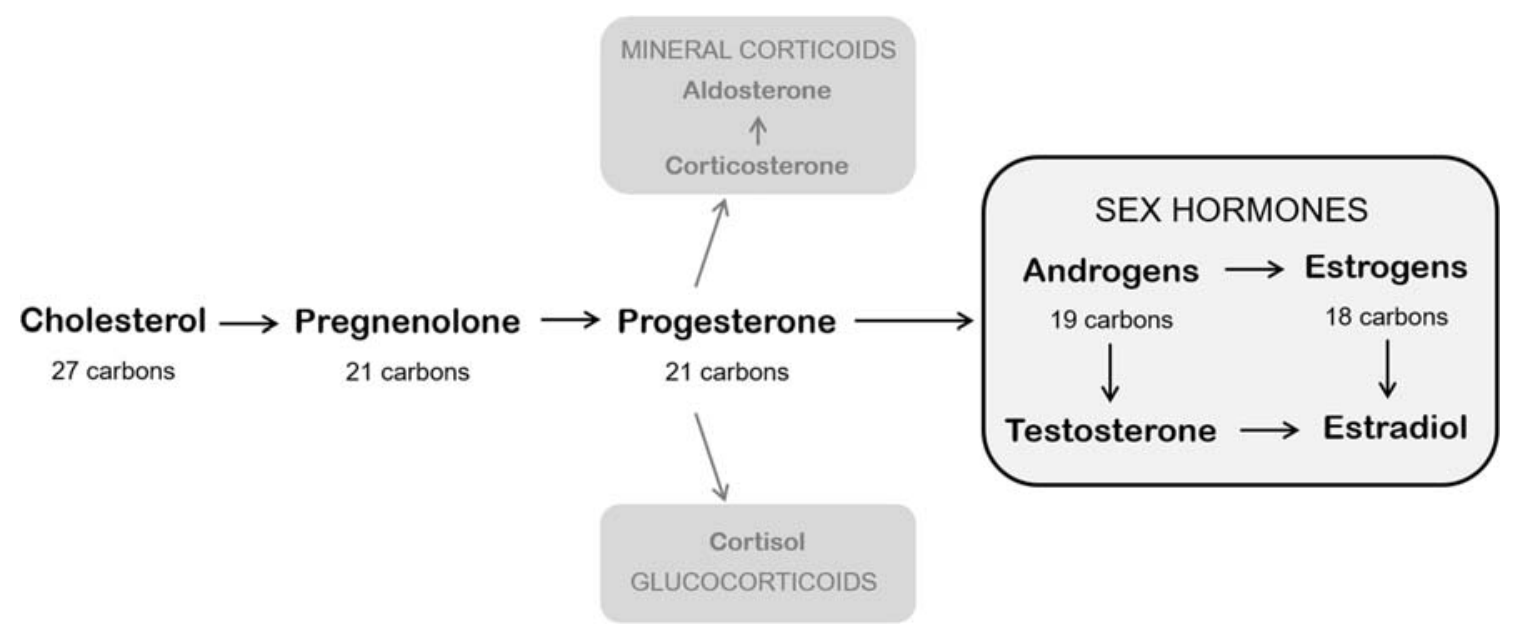

Fig. 1 Adapted from Frick et al. (2015). Simplified schematic representation of the steroid hormone biosynthesis pathway. Cholesterol is the essential precursor for all steroid hormones. Progesterone is the metabolic

functions (such as working memory and response inhibition) are particularly affected by estrogen (Colzato et al. 2010; Colzato et al. 2012; Hidalgo-Lopez and Pletzer 2017).

\section{Testosterone}

Testosterone plays a relevant role in brain structure and cognitive functions (Bramen et al. 2012; Ciocca et al. 2016). As pointed out by Janowsky (2006), androgen receptors are selectively retraceable in key brain areas such as the hippocampus (Beyenburg et al. 2000) and the prefrontal cortex (Finley and Kritzer 1999), paramount structures for higher-order cognitive processes. Interestingly, studies in animal models have shown that gonadectomy (surgical removal of either the testes) selectively decreases acetylcholine in the hippocampus and anterior cingulate cortex (Nakamura et al. 2002) and temporarily declines the increase of dopamine innervation in the cingulate cortex of rats (Adler et al. 1999). Both neurotransmitters are crucial for learning and memory (Cools 2006; Hasselmo 2006) and seem to be key elements in explaining how testosterone affects cognitive processes.

Moreover, sex differences specifically on mental rotation have been partly attributed to prenatal androgen exposure (Miller and Halpern 2014). In fact, females with a high exposure to prenatal androgens during pregnancy, such as those with congenital adrenal hyperplasia (CAH, Puts et al. 2008) or females with a male twin (Tapp et al. 2011), show better mental rotation abilities than control females (Heil et al. 2011). These findings suggest that prenatal androgens can influence postnatal cognitive abilities (Miller and Halpern 2014). Nevertheless, it seems that the strongest influence of androgens in brain development appears at the critical developmental periods including pregnancy (Heil et al. 2011; Puts et al. 2008) during puberty (Bramen et al. 2012; Vuoksimaa et al. 2012) or midlife (Yonker et al. 2006). precursor of other endogenous steroids such as sex hormones (androgens and estrogens) and corticoids. The enzyme aromatase converts androgens in estrogens

\section{Supplementation of Sex Hormones}

The role of hormones in cognitive regulation can be explored more directly by looking at studies that correlate increases/ decreases in cognitive capacities with respective increases/ decreases in sex hormones within individuals. Within those, we can consider the special cases of men and women in hormone replacement therapy due to illness or to treat symptoms associated with menopause and of those transsexual men and women taking corrective hormones. Both cases provide insight into the causal mechanisms of how sex hormones affect cognition (Vidal et al. 2006).

Estrogen can be given to women for the treatment of conditions that diminish their endogenous estrogen levels, such as menopause (Asthana 2003) or in amenorrhea (absence of menstruation). A recent randomized controlled trial has shown estrogen replacement to improve verbal memory and executive control in athletes with menstrual dysfunction (oligomenorrhea/amenorrhea, Baskaran et al. 2017). Both males and females experience a decline in sex hormones with healthy aging, and for females, there is a steep gonadal hormone decline with reproductive senescence during midlife. Consistent with the theories of estrogen's effects on cognition, verbal fluency and memory show concurrent decline postmenopause and aging women (Ardila et al. 2011). Notably, though men and women have different baseline levels of verbal memory and fluidity, the rate of change of this skill is similar in aging men (Ardila et al. 2011). However, a recent study by McCarrey et al. (2016) indicated that men showed an accelerated cognitive decline on tests assessing mental status, visuospatial ability, and visuomotor speed, while no steeper decline in any of these cognitive processes was seen in women. Reduction in circulating estrogen (estradiol) has been hypothesized to contribute to the increased risk for Alzheimer's disease in females (AD; Asthana 2003): around two-thirds of 
those diagnosed with $\mathrm{AD}$ are women (Gao et al. 1998). Indeed, the depletion of gonadal steroids at menopause and the associated depletion of their neuroprotective actions might be involved in the aggravated female vulnerability to $\mathrm{AD}(\mathrm{Li}$ and Singh 2014). Even though the Women's Health Initiative Memory Study led by Shumaker et al. (2003) showed cognitive decline with estrogen replacement in postmenopausal women, a few observational and randomized controlled trials have indicated that hormone therapies may decrease the risk of $\mathrm{AD}$ and that its early use (just prior menopause) is protective and later use is either not protective or even harmful (see Maki 2013, for a review). Consistent with this idea, Girard et al. (2017) found hormone therapy at early postmenopause to enhance cognitive control prefrontal mechanisms. However, two clinical trials (the Kronos Early Estrogen Prevention Study (Gleason et al. 2015) and the Early versus Late Intervention Trial (Hodis et al. 2015) examined early use of hormone therapies in healthy postmenopausal females and found no significant benefits. It is important to note, as suggested by Gurvich et al. (2018), that the effectiveness of hormone therapy might depend on the neural integrity status of individuals. That is, estrogen's neuroprotective effects might take place only when neural networks are in good health. Also worth noting is that estrogen supplementation exhibits an inverted u-shaped effect relating cognitive performance and estrogen level, which is a common characteristic of cognitiveeffective supplements and drugs (Colzato 2017; Colzato and Arntz 2017). This means that there are levels which are too high or too low to produce a positive cognitive effect (or even maintain a positive condition) (Bayer et al. 2018).

Testosterone therapy is prescribed to males suffering from hypogonadism or other gonadotropic disorders and sometimes to the elderly due to the diminishing testosterone production (Ardila et al. 2011). Like with estrogen in women, testosterone therapy has shown to postpone cognitive deterioration in men in some cases (Miller and Halpern 2014). These cases exclude congenital hypogonadism - which is believed to be irreversible because of the associated organizational effects of prenatal testosterone deficiency (Slabbekoorn et al. 1999). Men with low testosterone have shown to score lower in visuospatial tests than the average man, while women with androgen-increasing hormone disorders score higher than the average woman in these tests (Ardila et al. 2011). Additionally, in taking supplemental testosterone, men do not only slow down visuospatial cognitive deterioration but also maintain better mathematical problem-solving abilities compared to those not taking testosterone supplements (Gouchie and Kimura 1991).

Finally, in transsexual women (born biologically male), exogenous estrogen produces an interesting, yet coherent effect. Before starting hormone therapy (which often includes androgen blockers), transgender women exhibit similar androgen levels as men, and (thus) they perform similarly to men, having greater visuospatial and lower verbal scores than women. Months after therapy, estrogen reaches levels similar to women's, and performance in verbal tasks increases, while their performance in visuospatial tasks decreases (Slabbekoorn et al. 1999). Similarly, in transsexual men (born biologically female), androgen hormone therapy increases visuospatial performance and lowers verbal memory and fluidity (Slabbekoorn et al. 1999). Along the same line, transsexual women reported superior verbal memory (Cohen-Kettenis et al. 1998) compared to same sex controls and improved immediate and delayed verbal memory after hormone therapy (Miles et al. 1998). Further, visuospatial ability has been found to be decreased in transsexual women when compared to same sex controls (van Goozen et al. 2002). However, two other studies found no effect of estrogen treatment on cognition in transsexual women (Miles et al. 2006; Wisniewski et al. 2005).

Even though the studies discussed in this paragraph seem to be promising and might qualify for the use of the two hormones as potential enhancers of cognition, it is important to keep in mind the failure to replicate some studies (Miles et al. 2006; Wisniewski et al. 2005) and that the supplementation of sex hormones is associated with undesirable side effects such as nausea/vomiting, stomach upset, or headache. That is, the side effects might overshadow the mild beneficial cognitive effects acquired from taking sex hormones as supplements.

\section{Conclusions}

There is mixed research showing group differences in cognitive abilities between men and women with the exception of relatively consistent findings showing superior visual abilities in males. These findings have been difficult to evaluate because it is hard to tease apart sociocultural gender differences from differences that can be attributed to biological sex differences including endogenous sex hormones. Even though some studies on patients receiving exogenous sex hormones revealed gender differences that emerge at group levels on a few cognitive tasks, it is not yet clear whether these differences can be partially attributed to hormonal causes.

Considering the mixed findings in the literature, at this point, for midlife associated with hormone decline with reproductive senescence and $\mathrm{AD}$, it is not possible to draw any definitive conclusions about whether estrogen seems to be able to maintain - or hold - levels of cognitive functioning, rather than enhancing them. Future studies need to shed light on the neurological underpinnings of the cognitive enhancement effects induced by sex hormones by investigating the neurochemistry of estrogens and testosterone without the confounding variables of diseases. As for the use of sex hormones as cognitive enhancers, the studies in transsexual individuals 
are more revealing, showing that the supplementation of female estrogen can enhance verbal skills whereas masculine androgen can increase performance in mathematical and visuospatial tasks. However, as pointed out in the previous section, also in this case, we cannot draw definitive conclusion because of some studies reporting null findings.

In sum, given the mixed findings in the literature and that more research is necessary, we cannot yet propose the supplementation of sex hormones as a promising tool for the maintenance rather than the enhancement of cognitive functions, especially to support neuroprotective functions across the life span.

Acknowledgements Partly of this work has been carried out when LSC, the corresponding author, was also affiliated to Leiden University.

\section{Compliance with Ethical Standards}

Conflict of Interest The authors declare that there they have no conflict(s) of interest.

Open Access This article is licensed under a Creative Commons Attribution 4.0 International License, which permits use, sharing, adaptation, distribution and reproduction in any medium or format, as long as you give appropriate credit to the original author(s) and the source, provide a link to the Creative Commons licence, and indicate if changes were made. The images or other third party material in this article are included in the article's Creative Commons licence, unless indicated otherwise in a credit line to the material. If material is not included in the article's Creative Commons licence and your intended use is not permitted by statutory regulation or exceeds the permitted use, you will need to obtain permission directly from the copyright holder. To view a copy of this licence, visit http://creativecommons.org/licenses/by/4.0/.

\section{References}

Adler, A., Vescovo, P., Robinson, J. K., \& Kritzer, M. F. (1999). Gonadectomy in adult life increases tyrosine hydroxylase immunoreactivity in the prefrontal cortex and decreases open field activity in male rats. Neuroscience, 89(3), 939-954.

Ardila, A., Rosselli, M., Matute, E., \& Inozemtseva, O. (2011). Gender differences in cognitive development. Developmental Psychology, 47(4), 984-990.

Asthana, S. (2003). Estrogen and cognition: The story so far. The Journals of Gerontology Series A: Biological Sciences and Medical Sciences, 58(4), M322-M323.

Ball, P., Knuppen, R., Haupt, M., \& Breuer, H. (1972). Interactions between estrogens and catecholamines. Studies on the methylation of catechol estrogens, catechol amines and other catechols by the catechoI-O-methyltransferase of human liver. Journal of Clinical Endocrinology and Metabolism, 34, 736-746.

Baskaran, C., Cunningham, B., Plessow, F., Singhal, V., Woolley, R., Ackerman, K. E., et al. (2017). Estrogen replacement improves verbal memory and executive control in oligo-amenorrheic athletes in a randomized controlled trial. The Journal of Clinical Psychiatry, $78(5)$, e 490.

Bayer, J., Gläscher, J., Finsterbusch, J., Schulte, L. H., \& Sommer, T. (2018). Linear and inverted u-shaped dose-response functions describe estrogen effects on hippocampal activity in young women. Nature Communications, 9(1), 1220.

Beyenburg, S., Watzka, M., Clusmann, H., Blümcke, I., Bidlingmaier, F., Elger, C. E., \& Stoffel-Wagner, B. (2000). Androgen receptor mRNA expression in the human hippocampus. Neuroscience Letters, 294(1), 25-28.

Bramen, J. E., Hranilovich, J. A., Dahl, R. E., Chen, J., Rosso, C., Forbes, E. E., Dinov, I. D., Worthman, C. M., \& Sowell, E. R. (2012). Sex matters during adolescence: Testosterone-related cortical thickness maturation differs between boys and girls. PLoS One, 7, e33850.

Ciocca, G., Limoncin, E., Carosa, E., Di Sante, S., Gravina, G. L., Mollaioli, D., et al. (2016). Is testosterone a food for the brain? Sexual Medicine Reviews, 4(1), 15-25.

Cohen-Kettenis, P. T., van Goozen, S. H. M., Doorn, C. D., \& Gooren, L. J. G. (1998). Cognitive ability and cerebral lateralisation in transsexuals. Psychoneuroendocrinology, 23, 631-641.

Colzato, L. S. (2017). Tyrosine. In L. S. Colzato (Ed.), Theory-driven approaches to cognitive enhancement. New York: Springer.

Colzato, L. S., \& Arntz, F. E. (2017). Ritalin. In L. S. Colzato (Ed.), Theory-driven approaches to cognitive enhancement. New York: Springer.

Colzato, L. S., \& Hommel, B. (2014). Effects of estrogen on higher-order cognitive functions in unstressed human females may depend on individual variation in dopamine baseline levels. Frontiers in Neuroscience, 8, 65 .

Colzato, L. S., Hertsig, G., van den Wildenberg, W., \& Hommel, B. (2010). Estrogen modulates inhibitory control in healthy human females: Evidence from the stop-signal paradigm. Neuroscience, 167, 709-715.

Colzato, L. S., Pratt, J., \& Hommel, B. (2012). Estrogen modulates inhibition of return in healthy human females. Neuropsychologia, 50, 98-103.

Cools, R. (2006). Dopaminergic modulation of cognitive functionimplications for L-DOPA treatment in Parkinson's disease. Neuroscience \& Biobehavioral Reviews, 30(1), 1-23.

Finley, S. K., \& Kritzer, M. F. (1999). Immunoreactivity for intracellular androgen receptors in identified subpopulations of neurons, astrocytes and oligodendrocytes in primate prefrontal cortex. Journal of Neurobiology, 40(4), 446-457.

Frick, K. M., Kim, J., Tuscher, J. J., \& Fortress, A. M. (2015). Sex steroid hormones matter for learning and memory: Estrogenic regulation of hippocampal function in male and female rodents. Learning \& Memory, 22(9), 472-493.

Gao, S., Hendrie, H. C., Hall, K. S., \& Hui, S. (1998). The relationships between age, sex, and the incidence of dementia and Alzheimer disease: A meta-analysis. Archives of General Psychiatry, 55(9), 809-815.

Girard, R., Météreau, E., Thomas, J., Pugeat, M., Qu, C., \& Dreher, J. C. (2017). Hormone therapy at early post-menopause increases cognitive control-related prefrontal activity. Scientific Reports, 7, 44917.

Gleason, C. E., Dowling, N. M., Wharton, W., Manson, J. E., Miller, V. M., Atwood, C. S., et al. (2015). Effects of hormone therapy on cognition and mood in recently postmenopausal women: Findings from the randomized, controlled KEEPS-cognitive and affective study. PLoS Medicine, 12(6), e1001833.

Gouchie, C., \& Kimura, D. (1991). The relationship between testosterone levels and cognitive ability patterns. Psychoneuroendocrinology, 16(4), 323-334.

Gurvich, C., Hoy, K., Thomas, N., \& Kulkarni, J. (2018). Sex differences and the influence of sex hormones on cognition through adulthood and the aging process. Brain Sciences, 8(9), 163.

Hara, Y., Waters, E. M., McEwen, B. S., \& Morrison, J. H. (2015). Estrogen effects on cognitive and synaptic health over the life course. Physiological Reviews, 95(3), 785-807.

Hasselmo, M. E. (2006). The role of acetylcholine in learning and memory. Current Opinion in Neurobiology, 16(6), 710-715. 
Heil, M., Kavšek, M., Rolke, B., Beste, C., \& Jansen, P. (2011). Mental rotation in female fraternal twins: Evidence for intra-uterine hormone transfer? Biological Psychology, 86, 90-93.

Hidalgo-Lopez, E., \& Pletzer, B. (2017). Interactive effects of dopamine baseline levels and cycle phase on executive functions: The role of progesterone. Frontiers in Neuroscience, 11, 403.

Hodis, H. N., Mack, W. J., Shoupe, D., Azen, S. P., Stanczyk, F. Z., Hwang-Levine, J., ... \& Henderson, V. W. (2015). Methods and baseline cardiovascular data from the early versus late intervention trial with estradiol testing the menopausal hormone timing hypothesis. Menopause (New York, NY), 22(4), 391.

Hyde, J. S. (2014). Gender similarities and differences. Annual Review of Psychology, 65, 373-398. https://doi.org/10.1146/annurev-psych010213-115057.

Hyde, J. S. (2016). Sex and cognition: Gender and cognitive functions. Current Opinion in Neurobiology, 38, 53-56.

Janowsky, J. S. (2006). Thinking with your gonads: Testosterone and cognition. Trends in Cognitive Sciences, 10(2), 77-82.

Kaufman, A. S., Kaufman, J. C., Liu, X., \& Johnson, C. K. (2009). How do educational attainment and gender relate to fluid intelligence, crystallized intelligence, and academic skills at ages 22-90 years? Archives of Clinical Neuropsychology, 24(2), 153-163.

Li, R., \& Singh, M. (2014). Sex differences in cognitive impairment and Alzheimer's disease. Frontiers in Neuroendocrinology, 35(3), 385403.

Luine, V. N. (2014). Estradiol and cognitive function: Past, present and future. Hormones and Behavior, 66(4), 602-618.

Majeres, R. L. (1997). Sex differences in phonetic processing: Speed of identification of alphabetical sequences. Perceptual and Motor Skills, 85(3_suppl), 1243-1251.

Majeres, R. L. (1999). Sex differences in phonological processes: Speeded matching and word reading. Memory \& Cognition, 27(2), 246-253.

Maki, P. M. (2013). The critical window hypothesis of hormone therapy and cognition: A scientific update on clinical studies. Menopause (New York, NY), 20(6), 695.

McCarrey, A. C., An, Y., Kitner-Triolo, M. H., Ferrucci, L., \& Resnick, S. M. (2016). Sex differences in cognitive trajectories in clinically normal older adults. Psychology and Aging, 31, 166-175.

Miles, C., Green, R., Sanders, G., \& Hines, M. (1998). Estrogen and memory in a transsexual population. Hormones and Behavior, 34, 199-208.

Miles, C., Green, R., \& Hines, M. (2006). Estrogen treatment effects on cognition, memory and mood in male-to-female transsexuals. Hormones and Behavior, 50(5), 708-717.

Miller, E. K. (2000). The prefrontal cortex and cognitive control. Nature Reviews Neuroscience, 1, 59-65.

Miller, D. I., \& Halpern, D. F. (2014). The new science of cognitive sex differences. Trends in Cognitive Sciences, 18(1), 37-45.

Milne, M. R., Haug, C. A., Ábrahám, I. M., \& Kwakowsky, A. (2014). Estradiol modulation of neurotrophin receptor expression in female mouse basal forebrain cholinergic neurons in vivo. Endocrinology, $156(2), 613-626$.

Nakamura, N., Fujita, H., \& Kawata, M. (2002). Effects of gonadectomy on immunoreactivity for choline acetyltransferase in the cortex, hippocampus, and basal forebrain of adult male rats. Neuroscience, 109(3), 473-485.
Pletzer, B., Scheuringer, A., \& Scherndl, T. (2017). Global-local processing relates to spatial and verbal processing: Implications for sex differences in cognition. Scientific Reports, 7(1), 10575.

Puts, D. A., McDaniel, M. A., Jordan, C. L., \& Breedlove, S. M. (2008). Spatial ability and prenatal androgens: Metaanalyses of congenital adrenal hyperplasia and digit ratio (2D:4D) studies. Archives of Sexual Behavior, 37, 100-111.

Scheiber, C., Reynolds, M. R., Hajovsky, D. B., \& Kaufman, A. S. (2015). Gender differences in achievement in a large, nationally representative sample of children and adolescents. Psychology in the Schools, 52(4), 335-348.

Scheuringer, A., Wittig, R., \& Pletzer, B. (2017). Sex differences in verbal fluency: The role of strategies and instructions. Cognitive Processing, 18(4), 407-417.

Shumaker, S. A., Legault, C., Rapp, S. R., Thal, L., Wallace, R. B., Ockene, J. K., et al. (2003). Estrogen plus progestin and the incidence of dementia and mild cognitive impairment in postmenopausal women: The Women's Health Initiative memory study: A randomized controlled trial. JAMA, 289(20), 2651-2662.

Sinclair, D., Purves-Tyson, T. D., Allen, K. M., \& Weickert, C. S. (2014). Impacts of stress and sex hormones on dopamine neurotransmission in the adolescent brain. Psychopharmacology, 231(8), 1581-1599.

Slabbekoorn, D., Van Goozen, S. H., Megens, J., Gooren, L. J., \& CohenKettenis, P. T. (1999). Activating effects of cross-sex hormones on cognitive functioning: A study of short-term and long-term hormone effects in transsexuals. Psychoneuroendocrinology, 24(4), 423-447.

Tapp, A. L., Maybery, M. T., \& Whitehouse, A. J. (2011). Evaluating the twin testosterone transfer hypothesis: A review of the empirical evidence. Hormones and Behavior, 60, 713-722.

Tuscher, J. J., Luine, V., Frankfurt, M., \& Frick, K. M. (2016). Estradiolmediated spine changes in the dorsal hippocampus and medial prefrontal cortex of ovariectomized female mice depend on ERK and mTOR activation in the dorsal hippocampus. Journal of Neuroscience, 36(5), 1483-1489.

van Goozen, S. H. M., Slabbekoorn, D., Gooren, L. J. G., Sanders, G., \& Cohen-Kettenis, P. T. (2002). Organizing and activating effects of sex hormones in homosexual transsexuals. Behavioral Neuroscience, 116, 982-988.

Vidal, A., Puig, O., Boget, T., \& Salamero, M. (2006). Gender differences in cognitive functions and influence of sex hormones. Actas Españolas de Psiquiatría, 34(6), 408-415.

Vuoksimaa, E., Kaprio, J., Eriksson, C. J., \& Rose, R. J. (2012). Pubertal testosterone predicts mental rotation performance of young adult males. Psychoneuroendocrinology, 37, 1791-1800.

Wisniewski, A. B., Prendeville, M. T., \& Dobs, A. S. (2005). Handedness, functional cerebral hemispheric lateralization, and cognition in male-to-female transsexuals receiving cross-sex hormone treatment. Archives of Sexual Behavior, 34(2), 167-172.

Yang, Y., \& Barth, J. M. (2015). Gender differences in STEM undergraduates' vocational interests: People-thing orientation and goal affordances. Journal of Vocational Behavior, 91, 65-75.

Yonker, J. E., Eriksson, E., Nilsson, L. G., \& Herlitz, A. (2006). Negative association of testosterone on spatial visualization in 35 to 80 year old men. Cortex, 42, 376-386.

Publisher's Note Springer Nature remains neutral with regard to jurisdictional claims in published maps and institutional affiliations. 